\title{
Treatment of acute intermittent porphyria during pregnancy and posterior reversible encephalopathy syndrome after delivery: A case report
}

\author{
JIAN ZHANG，YITING HU，JIMIN ZHENG，JUNCHA GAO，HONGTAO HOU, NA LIU and YUZHEN WANG \\ Department of Gastroenterology and Hepatology, Hebei General Hospital, Shijiazhuang, Hebei 050051, P.R. China
}

Received March 4, 2016; Accepted June 23, 2017

DOI: $10.3892 /$ etm.2017.5212

\begin{abstract}
Acute intermittent porphyria (AIP) is a rare inherited disorder of heme metabolism. It has the ability to trigger posterior reversible encephalopathy syndrome (PRES), a rare acute neurologic condition that is characterized by acute neurological symptoms. Pregnancy may induce AIP attacks. The present report describes the case of a pregnant woman with AIP. The patient was treated with heme-arginate during pregnancy and successfully delivered a healthy baby. Following delivery, the patient presented with PRES and experienced generalized seizures. Treatment including arginine hemoglobin, calcium gluconate and sodium chloride was administered. The symptoms of epilepsy did not recur. Magnetic resonance imaging of the head demonstrated that the bilateral occipital lobe lesions were significantly reduced in size following the treatment. In the present report, it was demonstrated that administration of heme-arginate for AIP during pregnancy is safe. Timely administration of arginine hemoglobin, calcium gluconate and sodium chloride may more efficiently improve the clinical status in AIP patients diagnosed with PRES who experience generalized seizures.
\end{abstract}

\section{Introduction}

Acute intermittent porphyria (AIP) is a rare inherited disorder of heme metabolism that mainly affects the nervous system, although only $10-15 \%$ of gene carriers exhibit the clinical symptoms $(1,2)$. The majority of patients with AIP experience acute attacks that manifest as a combination of autonomic dysfunction, abdominal pain and mild psychological symptoms (3). Pregnancy represents a notable risk factor in patients with AIP. The acute attacks associated with AIP have been reported as being more common during pregnancy (24-95\%), particularly

Correspondence to: Dr Yuzhen Wang, Department of Gastroenterology and Hepatology, Hebei General Hospital, 348 Heping West Road, Shijiazhuang, Hebei 050051, P.R. China E-mail: wangyuzhenm@163.com

Key words: acute intermittent porphyria, posterior reversible encephalopathy syndrome, arginine hemoglobin during the first trimester (4). AIP in pregnant women is also associated with higher rates of spontaneous abortion, hypertension, low birth weight and infant mortality (2-42\%) $(4,5)$. AIP may also trigger posterior reversible encephalopathy syndrome (PRES), a rare acute neurological condition that is characterized by acute neurological symptoms including seizures, visual abnormalities, headache, vomiting, nausea, impaired consciousness and focal neurologic deficits (6). It has previously been reported that the majority of pregnant PRES patients exhibited abrupt hypertension, in accordance with the theory that hyperperfusion induced by abrupt hypertension exceeding the retaining capacity of the brain capillary beds contributes to the development of PRES (7). Epileptic seizures are rare symptoms of AIP, occurring in $<10 \%$ of all patients with AIP (2). An exacerbated attack of AIP may occur during pregnancy due to hormonal changes (8). Delayed diagnosis and treatment of AIP in all patients may be fatal or result in permanent neurological damage (9). The present report describes the case of a pregnant woman with AIP who presented with PRES following cesarean delivery and was successfully treated with heme-arginate.

\section{Case report}

A 25-year-old woman presented at Hebei General Hospital (Shijiazhuang, China) on March 16, 2014 having experienced paroxysmal pain in the abdomen, abdominal distention, flatulence and coprostasis without acid reflux, heartburn, nausea, vomiting or fever for 10 days. The cousin of the patient was affected by AIP and had inherited it from her father. Examination revealed a high level of blood human chorionic gonadotropin $(551.50 \mathrm{IU} / \mathrm{ml}$; normal range, 50-500 IU/ml) 6 days post-menstrually. The urine of the patient became red following exposure to the sun for $0.5 \mathrm{~h}$. Porphyrin was detected in the urine, and the intracellular zinc porphyrin level was $10.3 \mu \mathrm{g} / \mathrm{gHb}$ (normal value, $0-4.7 \mu \mathrm{g} / \mathrm{gHb}$ ). A diagnosis of AIP and early pregnancy was made. Glucose $(14 \%, 500 \mathrm{ml} /$ day) was administered via intravenous drip for 12 days, with intravenous injection of esomeprazole (AstraZeneca, Cambridge, UK) $80 \mathrm{mg} / \mathrm{twice} /$ day for 8 days and a compound amino acid injection (18AA-II; Novamin; Fresenius Kabi Pharmaceutical Co., Ltd., Beijing, China) $250 \mathrm{ml} /$ day for 12 days. The abdominal pain was relieved significantly, and the patient was discharged from hospital 13 days after presentation. 
The patient presented at the hospital for a second time on July 12, 2014 at 20 weeks of gestation. She had felt continuous abdominal pain and dizziness during the previous 20 days, and her urine was red when exposed to light. These symptoms were considered to indicate an AIP attack. The patient was treated with intravenous injection of glucose $(14 \%, 500 \mathrm{ml} /$ day $)$ for 5 days, amino acid injection $250 \mathrm{ml} /$ day for 4 days, and a medium-chain and long-chain triglyceride lipid emulsion injection (20\%, $250 \mathrm{ml} /$ day; $\mathrm{C}_{8-24} \mathrm{Ve}$, Lipofundin MCT/LCT; B. Braun Melsungen, Melsungen, Germany) for 4 days. However, the symptoms remained. Following the provision of written informed consent by the patient, arginine hemoglobin (Normosang; Orphan Europe, Paris, France) was then administered to the patient for 4 consecutive days at a dose of $3 \mathrm{mg} / \mathrm{kg} /$ day as a slow intravenous infusion over $15 \mathrm{~min}$. The patient was also treated with serum albumin (40 g) and a leukocyte-reduced red blood cell suspension ( 6 units; both Hebei Blood Center, Shijiazhuang, China). The abdominal pain and dizziness eased significantly, and the patient was discharged 14 days after hospitalization.

The patient went to the hospital for a third time on August 31, 2014 having suffered from abdominal pain, nausea and vomiting for 4 days. Urine protein and urine bilirubin tests were positive. The patient was again treated with arginine hemoglobin, serum albumin and leukocyte-reduced red blood cell suspension as detailed above. The abdominal pain was eased significantly and the patient was discharged 28 days after hospitalization.

On October 3, 2014 the patient presented at the hospital for the fourth time. She had suffered abdominal pain with nausea and vomiting for 7 days. Arginine hemoglobin at a dose of $4 \mathrm{mg} / \mathrm{kg} /$ day over $15 \mathrm{~min}$ was administered for 2 consecutive days. The patient delivered a healthy baby boy by cesarean section in November 6, 2014. Two days later, the patient presented with headache, dizziness and blurred vision. Her blood pressure was $170 / 110 \mathrm{mmHg}$ and heart rate was 82 beats $/ \mathrm{min}$. A single dose of nitrendipine (10 mg; 39562-70-4; Shandong Tong Ren Pharmaceutical Co., Ltd., Linyi, China) was administered orally. Examination of the patient's head using magnetic resonance imaging (MRI), magnetic resonance angiography (MRA), magnetic resonance venography (MRV) and diffusion weighted imaging (DWI) led to a diagnosis of PRES due to the following observations: i) The lesion in the double occipital parietal cortex and subcortical exhibited gyrus-like or patchy signal; ii) long signals on T1W1 and T2W1; iii) high signals on fluid attenuation inversion recovery; iv) equal or slightly higher signals of DWI; v) high values of ADC; and vi) local shallow cerebral sulci. The patient was treated with intravenous injection of $250 \mathrm{ml} /$ day mannitol (Shijiazhuang No. 4 Pharmaceutical Co., Ltd., Shijiazhuang, China) for 7 days for dehydration to decrease the intracranial pressure. The patient then experienced seizures three times with blindness, and was treated with diazepam (Tianjin Jin Yao Amino Acid Co., Ltd., Tianjin, China) $10 \mathrm{mg}$ intravenous injection. As the patient's urine became red when exposed to light, arginine hemoglobin at a dose of $5 \mathrm{mg} / \mathrm{kg} / \mathrm{day}$ over 15 min was administered for 3 consecutive days. In addition, calcium gluconate $(1 \mathrm{~g} /$ day $)$, sodium chloride $(3 \%, 800 \mathrm{ml})$ and magnesium sulfate $(30 \mathrm{~g})$ were administered intravenously for 5 days. Examination using MRI (Fig. 1A-C), MRA, MRV and DWI was repeated $2 \mathrm{~h}$ after the epileptic episode, on the

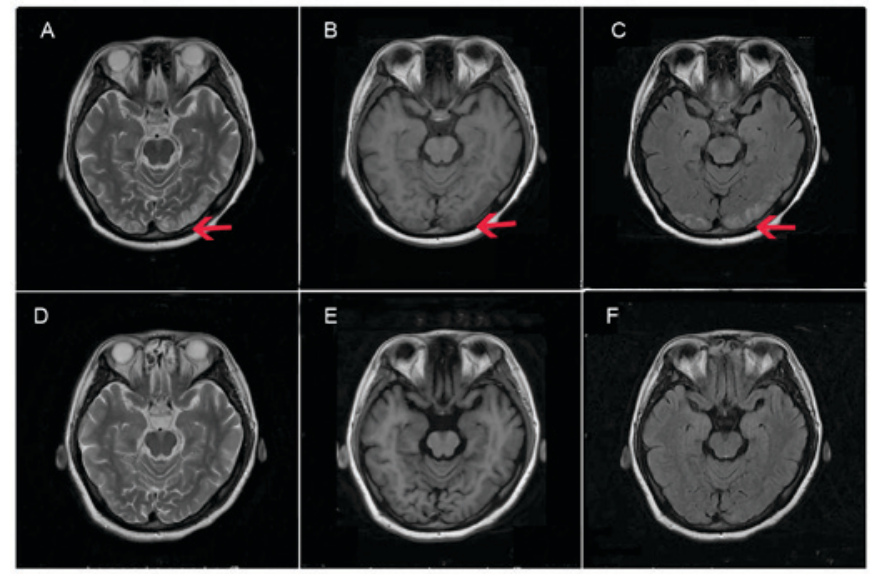

Figure 1. MRI findings performed at (A-C) $2 \mathrm{~h}$ after the epileptic episode, and (D-F) 10 days after admission. The lesion in double occipital parietal cortex and subcortical exhibited a gyrus-like or patchy signal. At $2 \mathrm{~h}$ after the epileptic episode, MRI revealed (A) long signal on T2W1 and local shallow cerebral sulci, (B) long signal on T1W1 and local shallow cerebral sulci, (C) high signal on FLAIR and local shallow cerebral sulci. MRI following the treatment exhibited (D) equal T1, (E) long T2 and (F) high FLAIR signals. These signals indicated the presence of cerebrocellular edema and the lesion area was reduced in size compared with that $2 \mathrm{~h}$ after the epileptic episode. Red arrows indicate the lesion area. MRI, magnetic resonance imaging; FLAIR, fluid attenuation inversion recovery.

day after the first imaging examination. The images revealed that the bilateral occipital lobe lesion had increased slightly in size. The treatment was continued and the patient's eyesight recovered gradually. No epileptic symptoms were exhibited. A third MRI examination, conducted 10 days after the second MRI, demonstrated that the bilateral occipital lobe lesions were significantly reduced (Fig. 1D-F). The patient was followed up with physical examinations to evaluate the presence of headache or abdominal pain, and to measure blood pressure, blood sodium and urine, prior to discharge. The condition of the patient improved significantly and she was discharged 12 days later.

\section{Discussion}

Pregnancy can induce acute attacks of AIP (10). There are very few drugs available for the treatment of AIP. The cornerstones of therapy are high-caloric infusions and treatment with heme-arginate (11). Treatment of patients with epilepsy may be challenging, as optimal antiepileptic treatment and heme-arginate therapy are not justified (12), due to the dysmorphogenetic and fetotoxic effects of heme-arginate. Epileptic seizures occurring during an acute AIP attack are very difficult to treat, since the majority of anticonvulsive agents actually promote AIP attacks (13).

In the present case, the patient was diagnosed with AIP when she presented at the hospital at 4 weeks of gestation. Heme-arginate treatment was used to control the symptoms of AIP. The patient successfully delivered a healthy baby. In the present case, the application of heme-arginate avoided the termination of pregnancy. Weinzierl et al reported the case of a pregnant woman who was not treated with heme-arginate and whose symptoms had not been controlled, where the pregnancy was terminated (14). 
PRES is a clinical entity characterized by temporary neurological symptoms, including acute headache, vision loss, altered mental status and coma (3). Porphyria is one of the etiological factors of PRES (15). MRI in patients with PRES exhibits posterior cerebral edema (16). Following the elimination of PRES-triggering factors and appropriate treatment, the clinical and radiological symptoms usually disappear (17).

Two theories have been proposed to explain the pathophysiology of PRES. One theory suggests that hypertension leads to failure of the autoregulation of blood flow, subsequent hyperperfusion and vasogenic edema, and the other suggests that vasoconstriction and hypoperfusion lead to brain ischemia and subsequent vasogenic edema (9). In the present case, high blood pressure was detected following delivery. The patient presented with PRES diagnosed by MRI at $48 \mathrm{~h}$ after childbirth. It is important to focus on the prevention of PRES in pregnant women with AIP, and the application of heme-arginate is suggested to be useful for the treatment of AIP-induced PRES. In the present patient, the treatment of seizures with diazepam appeared to be safe.

In summary, the present study indicated that the administration of heme-arginate for AIP during pregnancy was safe. The observations made in the present case suggest that the timely administration of heme-arginate, a high concentration of glucose, sodium chloride and magnesium sulfate may improve the clinical status of patients with AIP and PRES who experience generalized seizures.

\section{Acknowledgements}

The present study was supported by the Key Research Program of Science and Technology of Hebei Public Health Department (grant no. 20150115).

\section{References}

1. Aggarwal N, Bagga R, Sawhney H, Suri V and Vasishta K: Pregnancy with acute intermittent porphyria: A case report and review of literature. J Obstet Gynaecol Res 28: 160-162, 2002.

2. González-Arriaza HL and Bostwick JM: Acute porphyrias: A case report and review. Am J Psychiatry 160: 450-459, 2003.

3. Takata T, Kume K, Kokudo Y, Ikeda K, Kamada M, Touge T, Deguchi $\mathrm{K}$ and Masaki T: Acute intermittent porphyria presenting with posterior reversible encephalopathy syndrome, accompanied by prolonged vasoconstriction. Intern Med 56: 713-717, 2017.
4. Kantor G and Rolbin SH: Acute intermittent porphyria and caesarean delivery. Can J Anaesth 39: 282-285, 1992.

5. Farfaras A, Zagouri F, Zografos G, Kostopoulou A, Sergentanis TN and Antoniou S: Acute intermittent porphyria in pregnancy: A common misdiagnosis. Clin Exp Obstet Gynecol 37: 256-260, 2010.

6. Patil SV, Dhamangaonkar BR, Pattanshetti RC and Patil MM: Posterior reversible encephalopathy syndrome in early postpartum women: A case report. J Clin Diagn Res 8: RD01-RD02, 2014.

7. Wen Y, Yang B, Huang Q and Liu Y: Posterior reversible encephalopathy syndrome in pregnancy: A retrospective series of 36 patients from mainland China. Ir J Med Sci: Feb 2, 2017 (Epub ahead of print).

8. Kanaan C, Veille JC and Lakin M: Pregnancy and acute intermittent porphyria. Obstet Gynecol Surv 44: 244-249, 1989.

9. Anderson KE, Bloomer JR, Bonkovsky HL, Kushner JP, Pierach CA, Pimstone NR and Desnick RJ: Recommendations for the diagnosis and treatment of the acute porphyrias. Ann Intern Med 142: 439-450, 2005.

10. Armen TA, Sai-Sudhakar CB, Blais D and Awad H: Anesthetic management for combined double-valve and coronary artery bypass in a patient with acute intermittent porphyria. J Cardiothorac Vasc Anesth 23: 364-368, 2009.

11. Isenschmid M, König C, Fässli C, Haenel A, Hänggi W and Schneider H: Acute intermittent porphyria in pregnancy: Glucose or hematin therapy? Schweiz Med Wochenschr 122: 1741-1745, 1992 (In German).

12. Engelhardt K, Trinka E, Franz G, Unterberger I, Spiegel M, Beer R, Pfausler B, Kampfl A and Schmutzhard E: Refractory status epilepticus due to acute hepatic porphyria in a pregnant woman: Induced abortion as the sole therapeutic option? Eur J Neurol 11: 693-697, 2004

13. Sahu MT, Rajaram S, Saxena AK, Goel N and Ghumman S: Medical termination of pregnancy in acute intermittent porphyria. Gynecol Obstet Invest 62: 38-40, 2006.

14. Weinzierl A, Brezinka C and Engelhardt K: Unusual manifestation of acute hepatic porphyria in pregnancy. Fetal Diagn Ther 22: 136-138, 2007.

15. Lee VH, Wijdicks EF, Manno EM and Rabinstein AA: Clinical spectrum of reversible posterior leukoencephalopathy syndrome. Arch Neurol 65: 205-210, 2008.

16. Casey SO, Sampaio RC, Michel E and Truwit CL: Posterior reversible encephalopathy syndrome: Utility of fluid-attenuated inversion recovery MR imaging in the detection of cortical and subcortical lesions. AJNR Am J Neuroradiol 21: 1199-1206, 2000.

17. Tarim E and Giray S: Posterior reversible encephalopathy syndrome (PRES) in the differential diagnosis of eclampsia: Case report. Turkiye Klinikleri J Gynecol Obs 21: 213-215, 2011. 